\title{
Troponin in Chagas Disease
}

Roque Aras, Claudilson Bastos, Gildo Mota, Fábio Sodré, Agnaluce Moreira, Armando Tavares and José Carlos Lima
Federal University of Bahia, School of Medicine, Salvador, BA, Brazil
Chagas disease is still a major tropical disease in Latin America, affecting 16 to 18 million people. About 6 million people are infected with the causative organism, Trypanosoma cruzi, in Brazil [1]. However, it often remains for decades in its indeterminate form, symptomless, with tissue injury in about $30 \%$ of the cases, which eventually will evolve to serious arrhythmia and sudden death [1].

There is no effective clinical or laboratory technique to monitor chronic Chagas myocarditis. Several researchers have found that Troponin I and $\mathrm{T}$ are important biochemical markers of heart muscle damage. Increased levels of myocardial troponins have been found associated with acute myocardial ischemia, infarction, myocarditis and heart failure [2-4].

Recently, we tested sera from 60 Chagas disease patients ( 24 with the indeterminate form and 36 with chronic chagasic cardiomyopathy. Sera from 24 healthy volunteers (Control Group) were tested for Troponin I (Immulite 1000 Turbo DPCMedlab). The Troponin I value was considered normal when it was below $0.15 \mathrm{ng} / \mathrm{dl}$, and high when it was above $0.30 \mathrm{ng} / \mathrm{dl}$. The upper limit was set to be at least two standard deviations above the normal value.

The mean value for Troponin I was $0.46 \mathrm{ng} / \mathrm{dl}$ in the Chagas disease patients, and $0.027 \mathrm{ng} / \mathrm{dl}$ in the

Received on 17 July 2002; revised 22 September 2003. Address for correspondence: Dr..Andre C Lyra, MD, Serviço de Gastro-Hepatologia do Hospital São Rafael, Salvador-BA, Brasil. Rua Socrates Guanaes Gomes, 84, room 401, Salvador - Bahia - Brazil - Zip code: 40.283.320 Phone - Fax (55)-(71)452-9589. This study was supported in part by the CNPq.

The Brazilian Journal of Infectious Diseases 2003;7(6):358-359 (C) 2003 by The Brazilian Journal of Infectious Diseases and Contexto Publishing. All rights reserved. control group. The mean age was $44.1 \pm 9.9$ years of the Chagas patients, and 34 were male. When we tested We found $13(54 \%)$ and $26(74 \%)$ patients with high Troponin I, respectively, for chronic Chagas cardiomyopathy and the indeterminate form of Chagas disease. Twenty-one patients from the Chagas disease group were excluded due to other cardiovascular diseases, myopathy or kidney disease.

Troponin I levels were significantly higher among the Chagas disease patients with cardiomyopathy when compared to the indeterminate form and controls, mean $0.60 \mathrm{ng} / \mathrm{dl}$ vs $0.25 \mathrm{ng} / \mathrm{dl}$, respectively, and controls $0.027 \mathrm{ng} / \mathrm{dl}(\mathrm{P}<0.001)$.

All the patients with the indeterminate form of Chagas disease had normal EKGs, chest X-rays and echocardiograms. Possibly, the increased levels of Troponin I, found in our sample, are related to chronic foci of myocardial inflammation, provoked by Chagas disease.

Moreover, the utilization of a sensitive and easily measured biochemical marker should allow us to adopt different clinical cut-offs, facilitating the identification of the different degrees of myocardial damage, which now requires various diagnostic and therapeutic approaches [5].

The serum level of Troponin I is elevated in different clinical presentations of Chagas' disease and may become an important element for early detection of myocardial inflammation, to prevent further myocardial damage.

\section{References}

1. World Health Organization: Control of Chagas' disease, Report of a WHO. Geneva. World Health Organization Technical Series No. 811, 91p, 1991. 
2. Apple F.S. Cardiac Troponin I: in Cardiac Markers, Alan Wu. Ed. Humana Press Inc 1998; New Jersey, pp. 229-43.

3. La Vecchia L., Mezzena G., Zanolla L.,et al. Cardiac Troponin I as diagnostic and prognostic marker in severe heart failure. The Journal of Heart and Lung Transplantation 2000;19(7): 644-52.

4. Missov E., Calzolari C., Pau B. Circulating cardiac Troponin I in severe congestive heart failure. Circulation 1997;96(9):2953-8.

5. Lang K., Börner A., Figulla H.R. Comparison of biochemical markers for the detection of minimal myocardial injury: superior sensitivity of cardiac Troponin- T ELISA. Journal of Internal Medicine 2000;247:119-23. 